\title{
A Multilevel Analysis Method for Architecture Erosion
}

\author{
Tong Wang, Dongdong Wang, Bixin Li \\ School of Computer Science and Engineering \\ Southeast University, Nanjing, China
}

\begin{abstract}
During the evolution of software, improper change operations may cause architecture erosion. Architecture erosion creates problems on evolutionary costs, software performance and software quality. Many methods have been proposed to analyze architecture erosion. Architecture depends on the implementation of code, that is, architecture erosion is caused by code. However, few methods analyze the reasons for architecture erosion based on code. Besides, architecture is eroded with software evolution, but most methods do not analyze architecture erosion based on the change of software. In this paper, we propose a multilevel analysis method for architecture erosion. Our method contains three steps. Firstly, we detect the changed pairs based on two architectures by performing a multilevel change detection method. Secondly, we detect whether the corresponding code elements of changed pairs are erosion points by calculating erosion degree. Thirdly, we establish a cost-benefit model of repairing architecture erosion for repairing architecture erosion more effectively with repaired few erosion points. We illustrate our method through an open source project, and the experimental results indicate that our method can detect the erosion points of each level and the cost-benefit model is effectively.
\end{abstract}

Index Terms-architecture erosion, multilevel change detection, the cost-benefit model

\section{INTRODUCTION}

Software is continually evolved to meet new requirements. With the evolution of software, architecture erosion often occurs [1], [2]. Architecture erosion creates problems such as the increase of software evolutionary costs [3], the decrease of software performance [4], and the degradation of software quality [5]. In a word, uncontrolled architecture erosion has a negative impact on software [6]. In order to reduce the negative impacts, analyzing software architecture erosion in time is an important task during the development and maintenance of software [7].

Some researchers propose analysis methods for architecture erosion based on multiple types of indicators. Zhang et al. detect architecture erosion based on the design decision of architectural pattern [8], and Herold et al. detect architecture erosion based on a common ontology [7]. The purpose of detecting and repairing architecture erosion is reducing the effects on architecture quality [9], that is, the decrease of architecture quality is an important sign of architecture erosion, but few methods analyze architecture erosion based on quality. Besides, architecture depends on the implementation of source code [10], but few methods analyze architecture erosion based

B. Li is the corresponding author. E-mail: bx.li@seu.edu.cn DOI reference number:10.18293/SEKE2019-046 on code. Also, there may be many erosion points, but it is impossible to repair all erosion points, so how to reduce the erosion degree of architecture effectively by repairing fewer erosion points is an important problem.

To overcome the above limitations, we proposed the multilevel analysis method for architecture erosion. In our method, we first detect the changed pairs of each level by performing the multilevel change detection method. Then, we detect whether the corresponding code elements of changed pairs are erosion points by calculating the erosion degree. Finally, we establish the cost-benefit model for providing the repairing priority of erosion points.

To sum up, this work makes the following contributions.

- We detect the erosion points based on architecture quality which is an important sign of architecture erosion.

- We analyze reasons for architecture erosion based on the implementation of architecture by using the multilevel change detection method.

- We establish the cost-benefit model of repairing architecture erosion, in order to more effective repairing architecture erosion by repairing fewer erosion points.

This paper is organized as follows. Section II introduces the details of our method. In section III, we illustrate our method though an open source project. Section IV presents related work. In Section V, the threats to the validity of our method are analyzed. In Section VI, we make a conclusion and discuss future work.

\section{Our Method}

\section{A. Method Overview}

Architecture erosion leads to the gradual deterioration of software quality [11], that is, the deterioration of quality is an important sign of architecture erosion. So, in this paper, we define architecture erosion as follows.

Definition 1 architecture erosion: It is a phenomenon that occurs when architecture quality is decreased with software evolution.

The component dependency graph is a widely acceptable representation of architecture, in our method, we use it to represent architecture. The component dependency graph consists of the component and the dependency between components. A component is a code element of the high abstraction level, and it consists of multiple files. A file consists of multiple statements. According to the granularity of code elements, we 
divide architecture into three levels, component level, file level, and statement level.

The architecture contains multiple components. In an eroded architecture, not all components are eroded. Similarly, in an eroded component, not all files are eroded. And in an eroded file, not all statements are eroded. In order to narrow down the range of erosion for reducing the repairing cost, we analyze architecture erosion from architecture to component level, then from component level to file level, finally from file level to statement level.

Architecture is eroded with software evolution, so does components, files, and statements. In order to analyze whether components, files, and statements are eroded, we should first detect changed pairs of each level by using a multilevel change detection method. After detecting the changed pairs, we detect whether the corresponding code of changed pairs are eroded.

Definition 2 changed pair: Let $A 1$ be a original architecture. Let $A 2$ be a current architecture. Let $C 1$ and $C 2$ be two code elements of the above architectures. If $C 1$ is evolved to $C 2$, the two code elements constitute a changed pair. If $C 1$ is deleted and $C 2$ is a new code element, then they respectively constitute changed pairs with a virtual code element.

According to the above analysis, the overview of our method is shown in Figure 1. The first step is detecting the changed pairs of code elements, where add, delete and update operations are denoted in the green, red and blue border. The second step is calculating erosion degree to detect whether the corresponding code elements of changed pairs are erosion points, and the erosion points are denoted in gray nodes. The third step is establishing the relations between the number of repaired erosion points with the degree of decline in architecture erosion, that is, we establish a cost-benefit model of repairing erosion points. The model demonstrates how to reduce more erosion degree of architecture by repairing fewer erosion points.

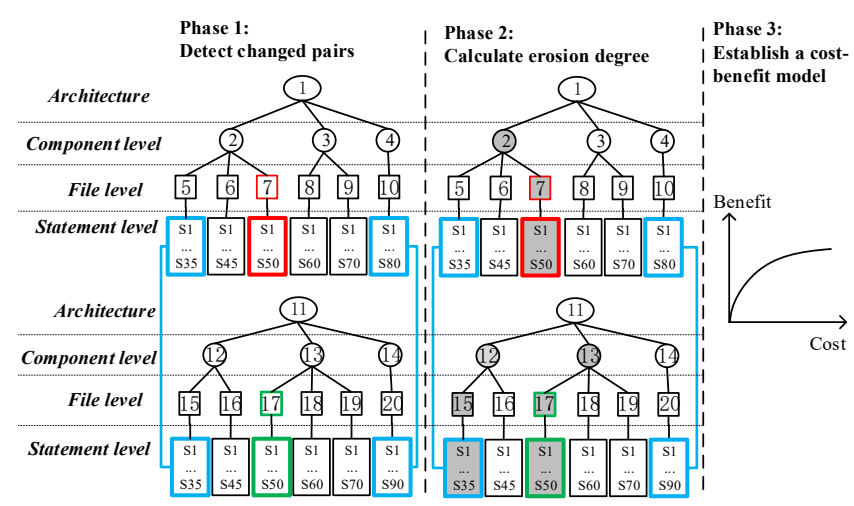

Fig. 1. An overview of our method.

\section{B. Detecting changed pairs of each level}

In order to detect changed pairs at file level and statement level, we perform the change detection method named the twostep multilevel program analysis tree matching method [12].
The method can detect multilevel changes effectively, such as method level, class level, and file level.

We perform the following steps to detect changed pairs at component level: calculating the similarity between components and identifying change operation based on component similarity.

The component similarity indicates the degree of similarity. The component consists of multiple files, so we calculate the component similarity based on the contained files. The formula is shown in Formula 1.

$$
\operatorname{Sim}(i, j)=\frac{2 *\left|S_{i} \bigcap S_{j}\right|}{L_{i}+L_{j}}
$$

where $\operatorname{Sim}(i, j)$ is the similarity between the $i_{t h}$ component and the $j_{t h}$ component, $S_{i}$ is the file set contained in the $i_{t h}$ component, $|X|$ is the number of elements of the set $X$ and $L_{i}$ is the number of code lines.

The components of the original architecture and the components of the current architecture are matched based on the component similarity to detect which components constitute a changed pair. We consider the following three change operations at the component level.

$\operatorname{Add}(C)$ : A new component $C$ is added in $V_{2}$.

Delete $(C)$ : In $V_{2}$, the component $C$ is deleted.

$\operatorname{Update}\left(C_{i}, C_{j}\right)$ : The component $C_{i}$ is updated to $C_{j}$.

In order to identify the above change operations based on the component similarity, we define two thresholds. The first threshold is HighThreshold. If the component similarity above HighThreshold, we deem that the two components are the same, that is, the component is not changed during the evolution process. The second threshold is LowThreshold. If the component similarity above HighThreshold but below LowThreshold, we deem that the change operation between the changed pair is updating. If the component similarity below LowThreshold, we deem that the two components of the changes pair are respectively the new component of the current architecture and the deleted component of the original architecture. The algorithm of identifying change operations is shown in Algorithm 1.

\section{Calculating erosion degree}

In this step, we detect whether the code elements of each changed pair are eroded, that is, whether the code elements are the erosion points.

Definition 3 erosion point: Let $A_{i}$ and $A_{j}$ constitute a changed pair which respectively belong to the original architecture and the current architecture. If the change operation between $A_{i}$ and $A_{j}$ causes architecture erosion, $A_{i}$ and $A_{j}$ are the erosion points.

The decrease in architecture quality is an important sign of architecture erosion. When architecture quality is decreased, it indicates that architecture is eroded, correspondingly, if architecture quality is increased, architecture is nor eroded.

In this paper, we take the change of understandability and testability as the indicators of architecture erosion. Understandability and testability are important quality attributes 


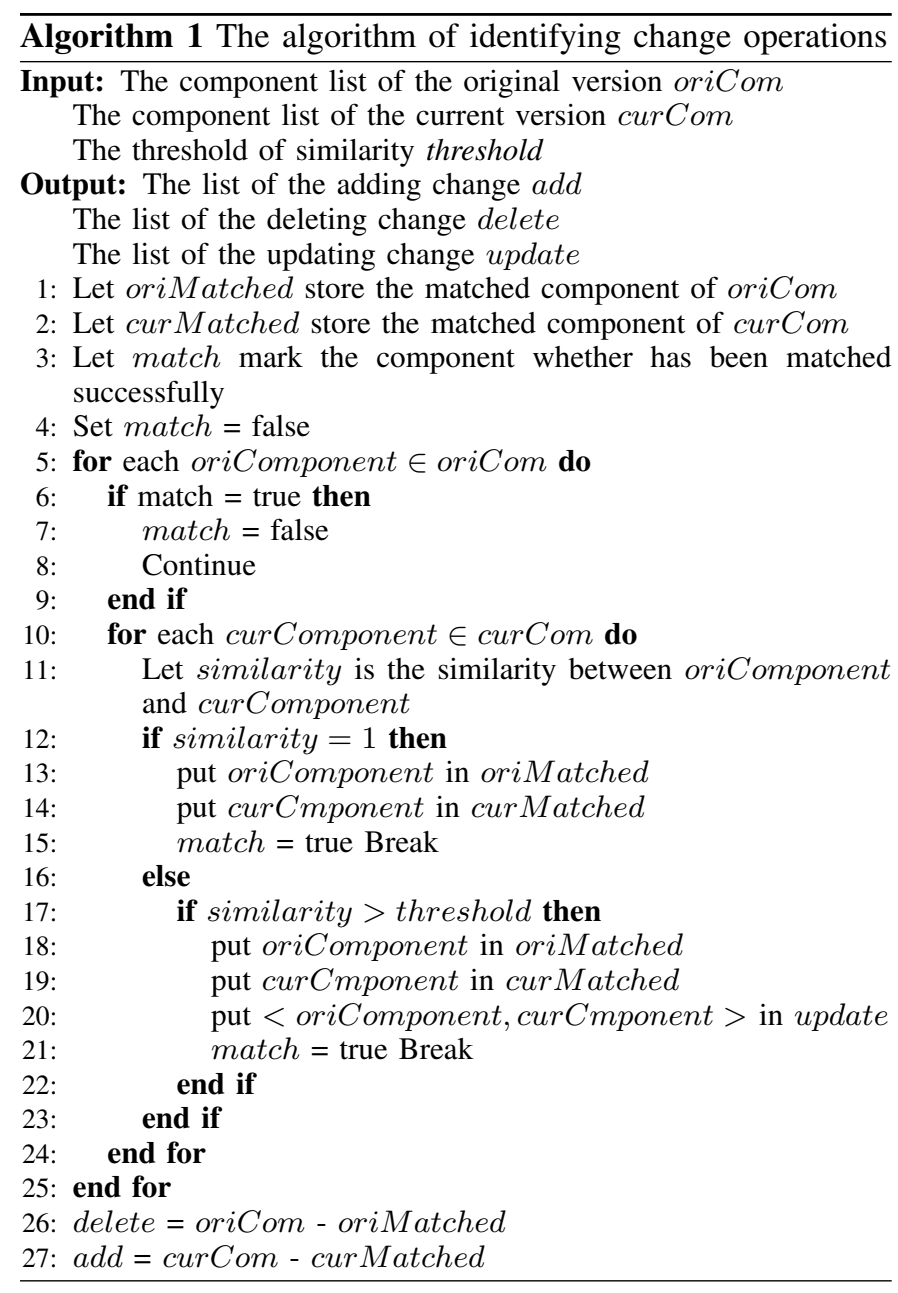

for architecture [13]. Firstly, we measure the two quality attributes.

Testability determines the required resources during testing and making test plans [14]. Its calculating formula is shown in Formula 2.

$$
\text { Testability }=1-\frac{\sum_{i=1}^{N} \frac{O_{i}}{N}}{N}
$$

where $T$ is the measurement of testability, $N$ is the number of elements, $i$ denotes the $i_{t h}$ code element, $O$ is the fanout of the $i_{t h}$ element.

Understandability denotes the difficulty of understanding architecture [15]. Its calculating formula is shown in Formula 3.

$$
\text { Understandability }=\frac{\sum_{i=1}^{N} f\left(C_{i}\right)}{N}
$$

where $U$ is the measurement of understandability, $N$ is the number of elements, $i$ denotes the $i_{t h}$ element, $L_{i}$ is number of code lines of the $i_{t h}$ element, $f\left(C_{i}\right)$ is the density of cognitive complexity of the code element $C_{i}$. The calculating formula of the density of cognitive complexity is shown in Formula 4.

$$
f\left(C_{i}\right)= \begin{cases}1 & \frac{C_{i}}{L_{i}} \leq 0.05 \\ 0.8 & 0.05<\frac{C_{i}}{L_{i}} \leq 0.1 \\ 0.6 & 0.1<\frac{C_{i}}{L_{i}} \leq 0.2 \\ 0.4 & 0.2<\frac{C_{i}}{L_{i}} \leq 0.3 \\ 0.2 & 0.3<\frac{C_{i}}{L_{i}} \leq 0.4\end{cases}
$$

where $f\left(C_{i}\right)$ is the density of cognitive complexity of the code element $C_{i}, L_{i}$ is number of code lines of the $i_{t h}$ element and $C_{i}$ is the cognitive complexity of the $i_{t h}$ element. Cognitive complexity can yield assessments of control flow that correspond to programmers intuitions about the mental, or cognitive effort required to understand those flows.

According to the above formulas, we the measurements of understandability and testability, then we use the erosion degree to represent the change of architecture quality before and after evolution.

Definition 4 erosion degree: Let $A_{o}$ and $A_{c}$ be architectures before and after software evolution. Let $A_{o}[i]$ be a code element of $A_{o}$ and $A_{c}[j]$ be a code element of $A_{c}$. If architecture quality is decreased, the difference between $A_{o}[i]$ and $A_{c}[j]$ is the erosion degree. If erosion degree is a negative value, it indicates that the changed pair does not damage architecture quality instead of increasing architecture quality.

The erosion degree is calculated based on a pair of code elements before and after software evolution. To architecture, the erosion degree depends on the change of the contained components. Similarity, to a component, the erosion degree depends on the changes of the contained files. Then, to file, the erosion degree depends on the changes of the contained statements.

The elements of the pair respectively belong to the original architecture and the current architecture. When a code element is deleted from the original architecture, and when a code element is added in the current architecture, there is not a corresponding code element in another architecture, so we use the average measurement as the threshold to calculate the erosion degree of the new code element and the deleted code element.

The formula of erosion degree is shown in Formula 5.

$$
f\left(E_{i}, E_{j}\right)= \begin{cases}M_{j}-M_{i} & E_{i} \text { is evolved to } E_{j} \\ M_{j}-A V G & E_{j} \text { is a new code element } \\ A V G-M_{i} & E_{i} \text { is a deleted code element }\end{cases}
$$

where $E_{i}$ is the $i_{t h}$ code element, $f\left(E_{i}, E_{j}\right)$ is difference between the measurements of $E_{i}$ and $E_{j}, M_{i}$ is the measurement of the $i_{t h}$ code element and $A V G$ is the average measurement of the previous architecture.

\section{Establishing the cost-benefit model}

Considering the number of erosion points and the repairing cost, in actual development process, it is impossible to repair all erosion points. In order to more effective repair architecture by repairing fewer erosion points, we establish the cost-benefit model based on erosion contribution. 
Definition 5 erosion contribution: Let $C$ be an erosion point. The erosion contribution of $C$ denotes that, compared with other eroded code elements of the same level, the degree of influence on architecture erosion.

The formula of the erosion contribution is shown in Formula 6.

$$
E C\left(P_{i}, C_{j}\right)=\frac{f\left(P_{i}, C_{i}\right)}{\sum_{(a, b) \in S}^{|S|} f\left(P_{a}, C_{b}\right)} * \operatorname{PEC}\left(P_{i}, C_{i}\right)
$$

where $O_{i}$ is the $i_{t h}$ code element of the original architecture, $C_{i}$ is the $i_{t h}$ element of the current architecture, $E C\left(O_{i}, C_{j}\right)$ is the erosion contribution of the changed pair constituted by $O_{i}$ and $C_{j}, f\left(O_{i}, C_{j}\right)$ is the erosion degree of the changed pair constituted by $O_{i}$ and $C_{j}, S$ is the set of changed pairs which are erosion points, $|S|$ is the number of the elements of $S,(a, b)$ is the element of $S$ and it denotes the changed pair constituted by $O_{a}$ and $C_{b}$, and $\operatorname{PEC}\left(P_{i}, C_{i}\right)$ is the erosion contribution of the changed pair which is the corresponding higher abstraction elements of $O_{i}$ and $C_{j}$. The component level is the highest abstraction level in our method. If $O_{i}$ is a component, the value of $\operatorname{PEC}\left(O_{i}, C_{i}\right)$ is 1 .

The higher erosion contribution indicates that the corresponding point has caused more negative effects on architecture. So the erosion contribution denotes the relative urgency degree of repairing. The order of repairing erosion points is based on the erosion contribution from high to low.

Unlike the erosion degree, the erosion contribution is a comparative value, because it is calculated based on the erosion of other code elements of the same level. The sum of erosion contribution of all erosion points is 1 . The sum of the erosion contribution of all repaired erosion points denotes the degree of repairing architecture erosion, so it is used as the indicator of the benefit.

Architecture depends on the implementation of the code, and in the actual development process, developers repair architecture erosion by modifying the code. So in our model, we only take the erosion points belonging to statement level into consideration.

Lines of code (LOC) is an important indicator for estimating software costs, so we use the number of repaired erosion points as the cost. With repairing erosion points, the erosion degree of architecture is reduced which is caused by repairing erosion points, so we use the sum of erosion contribution of the repaired erosion points as the benefit.

The erosion contribution denotes the degree of effects on architecture erosion. We sort all erosion points according to the erosion contribution from high to low. There is a relation between the number of repaired erosion points and the sum of erosion contribution, and the cost-benefit model is established based on the above relation.

\section{ILLUSTRATIVE EXAMPLE}

In this section, we choose an open source project as the case to illustrate the process of our method. commons-lang is a package which provides some basic APIs for some general operations, such as automatically generating toString () results, automatically implementing hashCode() and equals(), etc. commons - lang has received $1.5 \mathrm{~K}$ stars in GitHub.

We choose four versions as cases to detect architecture erosion. There is not a document about the architecture of commons-lang, so we perform the recovery method proposed by Kong et al. [16] to obtain its architecture. The basic information of commons-lang is shown in Table I, where the first column is the version number, the second column is the number of code lines, the third column is the measurement of testability, and the fourth column is the measurement of understandability.

TABLE I

THE BASIC INFORMATION OF COMMONS-LANG

\begin{tabular}{llll}
\hline Version & LOC & Testability & Understandability \\
\hline 3.1 .0 & $52.8 \mathrm{~K}$ & 0.800 & 0.760 \\
3.2 .0 & $61.6 \mathrm{~K}$ & 0.733 & 0.667 \\
3.3 .0 & $63.5 \mathrm{~K}$ & 0.834 & 0.667 \\
3.4 .0 & $66.0 \mathrm{~K}$ & 0.834 & 0.760 \\
\hline
\end{tabular}

Table I shows that, compared with the measurements of version 3.1.0, the measurements of version 3.2.0 are decreased. The decreasing measurements indicate that the architecture is eroded in the evolution process, so we analyze the evolution process. The erosion degree of understandability is -0.093 , and the erosion degree of testability is -0.067 .

The first step is detecting changed pairs. We implement the multilevel change detection method to detect the changed pairs of file level and statement level, and detect changed pairs of component level based on the component similarity. The number of changed code elements of each level is shown in Table II, where the first column is the change operation, where the second column to the fourth column are respectively component level, file level, and statement level. As the table shows that, there are six changed pairs at the component level, 220 changed pairs at the file level, and 6829 changed pairs at the statement level.

TABLE II

THE NUMBER OF CHANGED PAIRS OF EACH LEVEL

\begin{tabular}{lccc}
\hline \multirow{2}{*}{ Type } & \multicolumn{3}{c}{ The number of changed code elements } \\
\cline { 2 - 4 } & Component level & File level & Statement level \\
\hline Add & 1 & 29 & 3416 \\
Delete & 3 & 0 & 822 \\
Update & 2 & 191 & 1798 \\
Move & 1 & 0 & 803 \\
Total & 6 & 220 & 6839 \\
\hline
\end{tabular}

The second step is calculating erosion degree of each changed pairs. We first calculate the erosion degree of changed pairs of the component level, and the values of the erosion degree are shown in Table III, where the first column is the change operation of the component level, the second column is the erosion degree to testability, and the third column is the erosion degree to understandability. In the first column, we 
use update, add and delete to represent the change operation, and the corresponding element is the changed component. As shown in Table III, Add(src-1) has the highest erosion degree of testability, and Delete(commons $\backslash$ lang3-2) has the highest erosion degree of understandability.

TABLE III

THE EROSION DEGREE OF CHANGED PAIRS OF THE COMPONENT LEVEL

\begin{tabular}{lll}
\hline Change operation & ED_Test. & ED_Under. \\
\hline Update(commons $\backslash$ lang3) & 2.380 & -2.400 \\
Update(src) & -0.571 & -5.700 \\
Delete(commons $\backslash$ lang3-1) & 0.142 & 4.100 \\
Delete(commons $\backslash$ lang3-2) & -0.570 & 3.950 \\
Delete(lang3 $\backslash$ text) & -0.714 & 1.850 \\
Add(src-1) & 0.333 & -0.800 \\
\hline
\end{tabular}

There are 220 changed pairs of file level and 6839 changed pairs of statement level. Due to the limitation of space, we only show the erosion degree of some changed pairs in Table IV, where the first column is the change operation of file level, the second column is the erosion degree of testability, and the third column is the erosion degree of understandability. As shown in Table IV, Update(TypeUtils.java) has the highest erosion contribution to testability, Add(Conversion.java) has the highest erosion contribution to understandability.

TABLE IV

THE EROSION DEGREE OF CHANGED PAIRS OF FILE LEVEL

\begin{tabular}{lll}
\hline Change operation & ED_Test. & ED_Under. \\
\hline Add(Conversion.java) & 0 & 0.363 \\
Add(InheritanceUtils.java) & 0 & 0.233 \\
Update(CharSequenceUtils.java) & 0 & 0.215 \\
Update(StrSubstitutor.java) & 0 & 0.114 \\
Update(FastDateFormatTest.java) & 0 & 0.112 \\
Update(TypeUtils.java) & 0.376 & 0 \\
Update(FastDateFormat.java) & 0.233 & 0 \\
ADD(TypeLiteral.java) & 0.108 & -0.057 \\
ADD(FastDatePrinter.java) & 0.108 & 0.087 \\
ADD(Triple.java) & 0.108 & 0.028 \\
ADD(NotImplementedExceptionTest.java) & 0.067 & -0.086 \\
Update(StringUtilsEqualsIndexOfTest.java) & 0 & -0.009 \\
\hline
\end{tabular}

The third step is establishing the cost-benefit model based on erosion contribution. There are 6839 changed pairs of statement level, according to the erosion degree of them, we find that, 1027 changed pairs are erosion points for testability and 1453 changed pairs are erosion points for understandability. We sort these erosion points according to the erosion contribution from high to low. We respectively numbered them from 1 to 1027 , and from 1 to 1453 , then we establish the cost-benefit model. Due to the limitation of space, we only demonstrate the cost-benefit model of testability in Figure 2, where the x-axis represents the number of repaired erosion points, and the y-axis represents the sum of erosion contribution of the corresponding repaired erosion points.

As Figure 2 shows that, the growth rate of the sum of erosion contribution declines with repairing more erosion points, that is, the average benefit is reduced gradually. In

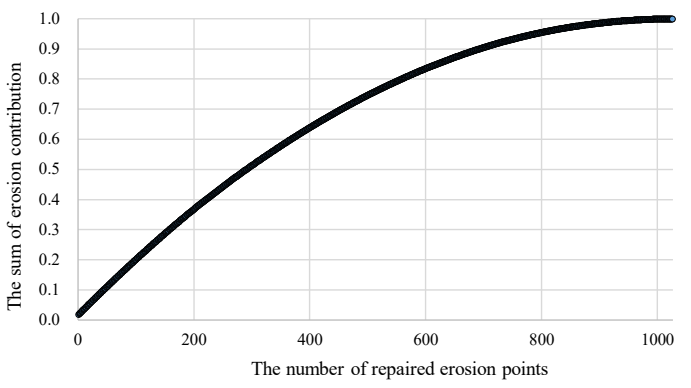

Fig. 2. The cost-benefit model of testability.

the example, we follow the Pareto principle which states that $80 \%$ of the effects come from $20 \%$ of the causes, so we only repair the top $20 \%$ erosion points.

We repair erosion points by rolling back them to their corresponding version. After rolling back the architecture is denoted as the new architecture. We detect erosion degree again based on the 3.1.0 version and the new architecture. The comparison of architecture quality is shown in Figure 3.

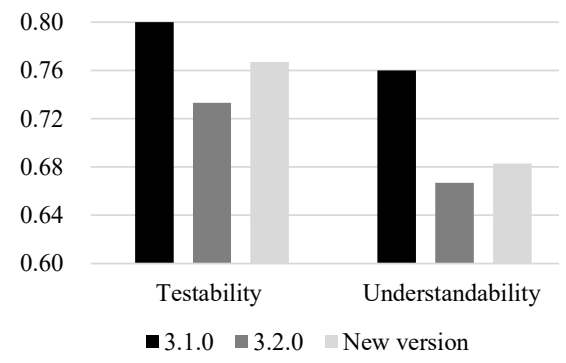

Fig. 3. The comparison of architecture quality.

According to Figure 3, we can draw two conclusions. First, compared with the version 3.2.0, the contribution degree is decreased, so the erosion points are detected and repaired. Second, only $20 \%$ erosion points are repaired, but the erosion degree falls by over $25 \%$, so the cost-benefit model proposes the repairing priority effectively.

\section{RELATED WORK}

The purpose of analyzing architecture erosion is how to locate the erosion points and how to repair the erosion points [10], so we compare our method with related work from the above aspects.

In the aspect of locating the erosion points, most researches propose analysis methods for architecture erosion based on one certain version. There are two main types of indicators, the inconsistency between the requirements and the actual implementation and the improper architecture pattern. Medvidovic et al. use the inconsistency as the indicator [17]. Zhang et al. detect architecture erosion of architectural pattern by the design decision [8]. Herold et al. detect architecture erosion of architecture pattern by the common ontology [7]. However, the above effects of architecture erosion all threaten the architecture quality [7], that is, avoiding the effects on 
software quality is the ultimate goal. So, in our method, we use the architecture quality as the indicators to detect architecture erosion.

In the aspect of repairing the erosion points. Terra et al. provide an approach to provide recommendations for removing architectural violations detected by the dependency constraint language [10]. Mair et al. propose a heuristic search method for adequate repairs using formalized and explicit knowledge of software engineers [9]. However, the actual architecture is implemented by its source code [10], so architecture erosion need be repaired by modifying code. So, our method analyzes architecture erosion from multiple levels.

In addition to the above aspects, our method establishes the cost-benefit model for repairing architecture erosion. In order to provide the repairing priority.

\section{THREATS TO VALIDITY}

Construct validity. We choose the component dependency graph as the representation of software architecture. Due to the limitations of the documents of architecture, we obtain architecture by implementing an architecture recovery method. There may be some deviations caused by the limitations of the recovery method. However, the recovery method has high accuracy, and in the future, the recovery method can be replaced by other better methods.

Internal validity. In our example, we roll back erosion points to their previous version. The experimental results indicate that, after rolling back, architecture erosion is repaired. However, rolling back erosion points may be not effective for maintaining functionality. However, the cost-benefit model is still useful for providing the priority of repairing erosion points.

External validity. In this paper, we choose testability and understandability as the indicators to detect architecture erosion. The two quality are quantified based on the attributes of components and dependencies, so we can use other quality as the indicators if it is quantified based on the features of components and dependencies.

\section{CONCLUSION AND FUTURE WORK}

In this paper, we propose a multilevel method for analyzing architecture erosion. Firstly, we detect changed pairs of each level by calculating component similarity and performing a multilevel change detection method. In this step, the changed code elements are matched. Secondly, we detect which changed pairs are the erosion points by calculating erosion degree. Thirdly, we establish the cost-benefit model of repairing architecture erosion based on the erosion contribution of each erosion point. The cost-benefit model is useful to obtain more benefits with less cost. We illustrate the process of our method through an example, and the experimental results indicate that our method can detect erosion points and the costbenefit model is useful for more effective repairing architecture erosion by repairing fewer erosion points.

In future work, we will investigate how to propose more suggestions for repairing architecture instead of rolling back.

\section{ACKNOWLEDGEMENTS}

This work is supported in part by the National Key R\&D Program of China under Grant 2018YFB1003902, in part by the Cooperation Project with Huawei Technologies Co., Ltd., under Grant YBN2016020009, and in part by National Natural Science Foundation of China under Grant 61872078, Grant 61572126, and Grant 61402103.

\section{REFERENCES}

[1] David Faitelson, Robert Heinrich, and Shmuel Tyszberowicz. Supporting software architecture evolution by functional decomposition. In International Conference on Model-Driven Engineering and Software Development, pages 435-442, 2017.

[2] Pooyan Behnamghader, Duc Minh Le, Joshua Garcia, Daniel Link, Arman Shahbazian, and Nenad Medvidovic. A large-scale study of architectural evolution in open-source software systems. Empirical Software Engineering, 22(3):1-48, 2016.

[3] Hongyu Pei Breivold and Ivica Crnkovic. A systematic review on architecting for software evolvability. In Australian Software Engineering Conference, pages 13-22, 2010.

[4] Fabian Brosig, Philipp Meier, Steffen Becker, Anne Koziolek, Heiko Koziolek, and Samuel Kounev. Quantitative evaluation of model-driven performance analysis and simulation of component-based architectures. IEEE Transactions on Software Engineering, 41(2):157-175, 2015.

[5] Ricardo Britto, Darja Smite, and Lars Ola Damm. Software architects in large-scale distributed projects: An ericsson case study. IEEE Software, 33(6):48-55, 2016.

[6] Santonu Sarkar, Shubha Ramachandran, G. Sathish Kumar, Madhu K. Iyengar, K. Rangarajan, and Saravanan Sivagnanam. Modularization of a large-scale business application: A case study. IEEE Software, 26(2):28-35, 2009.

[7] S Herold and A Rausch. Complementing model-driven development for the detection of software architecture erosion. In Proceedings of the 5th International Workshop on Modeling in Software Engineering, pages 24-30, 2013.

[8] Hui Song Franck Chauvel Hong Mei Lei Zhang, Yanchun Sun. Detecting architecture erosion by design decision of architectural pattern. In SEKE, pages $758-763,2011$

[9] Matthias Mair and Sebastian Herold. Towards extensive software architecture erosion repairs. In European Conference on Software Architecture, pages 299-306, 2013.

[10] Ricardo Terra, M. T. Valente, Krzysztof Czarnecki, and R. S. Bigonha. Recommending refactorings to reverse software architecture erosion. In European Conference on Software Maintenance \& Reengineering, pages 335-340, 2012.

[11] Lakshitha De Silva and Dharini Balasubramaniam. Controlling software architecture erosion: A survey. Journal of Systems \& Software, 85(1):132-151, 2012.

[12] Wang Tong, Wang Dongdong, Zhou Ying, and Li Bixin. Software multiple-level change detection based on two-step mpat matching. In IEEE International Conference on Software Analysis, Evolution and Reengineering, pages 4-14, 2019.

[13] Srdjan Stevanetic and Uwe Zdun. Exploring the relationships between the understandability of components in architectural component models and component level metrics. In International Conference on Evaluation \& Assessment in Software Engineering, pages 1-10, 2014.

[14] Samar Mouchawrab, Lionel C Briand, and Yvan Labiche. A measurement framework for object-oriented software testability. Information and software technology, 47(15):979-997, 2005.

[15] Jitender Kumar Chhabra, KK Aggarwal, and Yogesh Singh. Code and data spatial complexity: two important software understandability measures. Information and software Technology, 45(8):539-546, 2003.

[16] Xianglong Kong, Bixin Li, Lulu Wang, and Wensheng Wu. Directorybased dependency processing for software architecture recovery. IEEE Access, 6:52321-52335, 2018.

[17] Gruenbacher P Medvidovic N, Egyed A. Stemming architectural erosion by coupling architectural discovery and recovery. In Proceedings of the 2nd International Software Requirements to Architectures Workshop, pages 61-68, 2003. 